\title{
Reasons for the High Cesarean Delivery Rate among Women with Ankylosing Spondylitis: Using the Korean National Health Insurance Database
}

\author{
Jung Sun Lee, Ji Seon Oh, Ye-Jee Kim, Seokchan Hong (D), Chang-Keun Lee, Bin Yoo, \\ and Yong-Gil Kim
}

ABSTRACT. Objective. Women with ankylosing spondylitis (AS) have reported a higher rate of cesarean births
than healthy controls. This study aimed to identify factors associated with cesarean births in women
with AS.
Methods. Based on the Korean Health Insurance Review and Assessment Service claims database,
the subjects comprised female patients aged $20-49$ years old with AS. In total, 1293 deliveries after
AS diagnosis were included. A logistic regression analysis was performed to identify factors associated
with cesarean births.
Results. Among the 1293 deliveries in women with AS, 657 were cesarean and 636 were vaginal
deliveries. Compared to vaginal delivery, the women who had cesarean deliveries were older, had a
longer disease duration, and had a higher portion of primipara and dispensation of drugs. These factors
were associated with a higher risk of cesarean delivery: maternal age (OR 1.08, 95\% CI 1.04-1.12),
disease duration (OR 1.09, 95\% CI 1.03-1.14), and preeclampsia (OR 3.94, 95\% CI 1.17-13.32).
Further, compared to no drug dispensation, these drugs showed higher risks of cesarean delivery:
nonsteroidal antiinflammatory drugs (NSAID; OR 1.64, 95\% CI 1.31-2.37), tumor necrosis factor
inhibitor (TNFi), disease-modifying antirheumatic drugs (DMARD), or corticosteroids (OR 2.01,
95\% CI 1.57-2.58). In the subgroup analysis in primiparas, maternal age, or dispensation of NSAID
alone, or TNFi, DMARD, or corticosteroids was associated with a higher risk of cesarean delivery.
Conclusion. Women with AS showed a higher cesarean delivery rate, influenced by both maternal
age and disease-related factors. (First Release February 15 2020; J Rheumatol 2020;47:668-73;
doi:10.3899/jrheum.190754)

Key Indexing Terms:

ANKYLOSING SPONDYLITIS PREGNANCY DELIVERY CESAREAN BIRTH

The cesarean delivery rate has increased in many countries during recent decades ${ }^{1}$. Increasing maternal age, a higher proportion of previous cesarean deliveries, maternal preference for cesarean delivery, and increasing numbers of women with obesity or preexisting diseases are known risk

From the Division of Rheumatology, Department of Internal Medicine, University of Ulsan College of Medicine, and the Department of Biomedical Informatics, and the Department of Clinical Epidemiology and Biostatistics, Asan Medical Center, Seoul, Korea.

Supported by a grant from the Asan Institute for Life Sciences, Asan Medical Centre, Korea [2019-772].

J.S. Lee, MD, Department of Rheumatology, Asan Medical Center; J.S. Oh, MD, PhD, Department of Biomedical Informatics, Asan Medical Center; Y.J. Kim, PhD, Department of Clinical Epidemiology and Biostatistics, Asan Medical Center; S. Hong, MD, PhD, Department of Rheumatology, Asan Medical Center; C.K. Lee, MD, PhD, Department of Rheumatology, Asan Medical Center; B. Yoo, MD, PhD, Department of Rheumatology, Asan Medical Center; Y.G. Kim, MD, PhD, Department of Rheumatology, Asan Medical Center. Dr. J.S. Lee and Dr. J.S. Oh contributed equally to this study.

Address correspondence to Dr. Y.G. Kim, Division of Rheumatology, University of Ulsan College of Medicine, Asan Medical Center,

88, Olympic-ro 43-gil, Songpa-gu, Seoul 05505, Korea.

E-mail:bestmd2000@amc.seoul.kr

Accepted for publication September 27, 2019. factors for cesarean birth $2,3,4$. In South Korea, the cesarean delivery rate was $38 \%$ in 2015 , which is the fourth highest among Organisation for Economic Cooperation and Development countries ${ }^{5}$. This high rate is associated with older maternal age, improvement of socioeconomic status, and maternal obesity ${ }^{6}$.

Previous studies have shown that women with rheumatic diseases have a higher cesarean delivery rate ${ }^{7,8}$. Women with rheumatoid arthritis (RA) have a higher risk of cesarean delivery, and it is more common in patients with moderate to high disease activity ${ }^{9}$. Interestingly, the risk of primary cesarean delivery is higher in women with RA, but the risk factors for primary cesarean delivery do not differ compared to women without $\mathrm{RA}^{10}$. Further, elective cesarean delivery is twice as frequent among patients with chronic inflammatory arthritis, which could reflect maternal or obstetrician preference $^{8}$.

Ankylosing spondylitis (AS) affects the sacroiliac joints and commonly develops during reproductive age. Although the sacroiliac joint does not cause a mechanical hindrance during the delivery process, the rate of cesarean delivery was higher in women with AS than in healthy populations in

Personal non-commercial use only. The Journal of Rheumatology Copyright $\odot$ 2020. All rights reserved. 
previous studies ${ }^{11,12}$. The reason for the high rate of cesarean delivery in women with AS is unclear; however, women with AS are more likely to be exposed to drugs, to be older, and to have a higher prevalence of comorbidities or pregnancy complications including preterm birth than the general population ${ }^{11}$. Therefore, our present study aimed to identify factors related to AS that might have contributed to the choice of that delivery method in women with AS, using the Korean Health Insurance Review and Assessment Service (HIRA) claims database.

\section{MATERIALS AND METHODS}

Data sources. We conducted our study using patient records extracted from the HIRA claims database. The HIRA includes all health-related information on about 50 million individuals in the entire South Korean population covered by the National Health Insurance (NHI) program. It contains information on patient demographics, diagnosis [using the International Classification of Diseases, 10th revision (ICD-10)], medical procedures, prescriptions, and rare intractable disease (RID) registration information ${ }^{13}$. The prescription data include the generic name of the drug according to the HIRA drug formulary code, prescription date, days of supply, and route of administration. Since 2006, the NHI has operated a registration system for 133 RID, including AS. In the Korean RID system, a diagnosis is based on uniform diagnostic criteria distributed by the NHI and carefully reviewed by the corresponding healthcare institution and the NHI before registration. Study design and study subjects. The study cohort included female patients with AS aged 20-49 years who gave birth between July 1, 2007, and June 30, 2017. Patients who had been diagnosed with AS (ICD-10, M45) and had the RID code (V140) were included. All deliveries in the HIRA after the first date of AS diagnosis were included. The following data were collected from the HIRA database: demographic information, including age at delivery and diagnosis of AS, and whether the mother had experienced preterm birth, preeclampsia, twin pregnancy, or was a primipara; and comorbid medical conditions, including hypertension (HTN), diabetes mellitus, uveitis, inflammatory bowel disease (IBD), and psoriasis. Each delivery was categorized by the method of delivery: vaginal delivery or cesarean. To compare the cesarean delivery rate with women with RA or the general population, delivery records in women 20-49 years old who had been diagnosed with RA (ICD-10, M05) and women 20-49 years old without AS or RA during the study period were also evaluated.

Medications for AS. AS medications 12 months prior to delivery included oral nonsteroidal antiinflammatory drugs (NSAID; aceclofenac, celecoxib, diclofenac, etodolac, ibuprofen, indomethacin, ketorolac, mefenamate, meloxicam, morniflumate, nabumetone, naproxen, nimesulide, piroxicam, and sulindac); oral corticosteroids (defrazacort, methylprednisolone, prednisolone, and triamcinolone), disease-modifying antirheumatic drugs (DMARD; methotrexate and sulfasalazine), and tumor necrosis factor inhibitors (TNFi; adalimumab, etanercept, infliximab, and golimumab). Based on the AS medication, the treatment pattern was divided into 3 categories: (1) no dispensation of drugs, (2) NSAID alone, and (3) TNFi, DMARD, or corticosteroids.

Statistical analyses. All statistical analyses were performed using SAS Enterprise Guide software (version 6.1, SAS Institute Inc.). The baseline characteristics are presented as numbers with percentages for the categorical variables and as means with SD for the continuous variables. Characteristics of the AS patients were analyzed, including age, comorbidities, and medications. The comorbidities were identified by the ICD-10 codes (Supplementary Table 1, available with the online version of this article) for each patient and included HTN, diabetes mellitus, renal insufficiency, uveitis, IBD, and psoriasis. A logistic regression analysis was performed to identify the factors associated with cesarean delivery. Variables that had a $p$ value of $<0.1$ in the univariate analysis were selected for the multivariable analysis, but a p value of $<0.05$ was otherwise considered statistically significant in all analyses. To exclude the possibility of confounding by parity, which might affect the delivery methods, we also performed a logistic regression analysis in the primiparas (subgroup analysis).

Ethical approval. This study fulfilled the ethical guidelines of the Declaration of Helsinki. This study was approved by the Institutional Review Board of the Asan Medical Center (number 2017-0431). The requirement for informed consent was waived because it used an existing database.

\section{RESULTS}

Baseline characteristics according to delivery methods. In the Korean HIRA claims database, there were 1293 delivery records for women with AS, 8297 for women with RA, and 4.2 million for the general population between July 1, 2007, and June 30, 2017. The rate of cesarean delivery was the highest in women with AS (50.8\%), followed by women with RA (44.1\%), and the general population (38.0\%).

Table 1 shows the baseline characteristics according to delivery methods in women with AS. Among the 1293 delivery records from 996 women with AS, there were 657 cesarean deliveries and 636 vaginal deliveries. The mean maternal age was higher in the women with cesarean deliveries than in women with vaginal deliveries $(32.54 \pm 3.34$ vs $31.85 \pm 3.33 \mathrm{yrs}, \mathrm{p}<0.001)$. Further, the disease duration was longer in the women with cesarean deliveries than in the women with vaginal deliveries $(4.42 \pm 2.48$ vs $3.93 \pm 2.24$ $y r s, p<0.001)$. The frequency of primipara was higher in the women with cesarean deliveries than in the women with vaginal deliveries [404 (61.5\%) vs $345(54.2 \%), \mathrm{p}=0.01$ ]. However, the incidences of twin pregnancy and comorbidities including uveitis, IBD, and psoriasis did not differ between the 2 groups. When the patients were divided into 3 groups according to the treatment pattern 12 months prior to delivery, the frequency of no dispensation of medication was higher in women with vaginal deliveries than in women with cesarean deliveries [382 (60.1\%) vs $300(45.7 \%)$, $\mathrm{p}<0.001$ ]. However, the proportions of NSAID alone and TNFi, DMARD, or corticosteroids were higher in women with cesarean deliveries than in women with vaginal deliveries. Further, $41.6 \%, 24.7 \%, 19.8 \%$, and $12.6 \%$ of women with cesarean deliveries were exposed to NSAID, corticosteroids, DMARD, or TNFi within 12 months prior to delivery, respectively. For vaginal deliveries, the corresponding frequencies were $30.8 \%, 17 \%, 14.6 \%$, and $8.3 \%$, respectively.

Clinical factors associated with cesarean delivery in women with $A S$. A logistic regression analysis was performed to evaluate the factors associated with cesarean delivery (Table 2). In the univariate analysis, maternal age, disease duration, being primiparous, preterm birth, preeclampsia, and the pattern of treatment were associated with cesarean delivery. In the multivariable analysis, maternal age was associated with a higher risk of cesarean delivery (OR $1.08,95 \%$ CI $1.04-1.12, \mathrm{p}<0.001)$. In addition, a longer disease duration (OR $1.09,95 \%$ CI $1.03-1.14, \mathrm{p}<0.001$ ), being primiparous (OR 1.49, 95\% CI 1.16-1.89, $\mathrm{p}<0.001$ ), and the presence

Personal non-commercial use only. The Journal of Rheumatology Copyright @ 2020 . All rights reserved. 
Table 1. Baseline characteristics according to delivery methods in women with ankylosing spondylitis.

\begin{tabular}{|c|c|c|c|}
\hline Characteristics & nal Delivery, $\mathrm{n}=636$ & Cesarean Delivery, $\mathrm{n}=657$ & $\mathrm{p}^{*}$ \\
\hline Maternal age, yrs & $31.85 \pm 3.33$ & $32.54 \pm 3.34$ & $<0.001$ \\
\hline $20 \leq 25$ & $7(1.1)$ & $5(0.8)$ & 0.01 \\
\hline$>25 \leq 30$ & $144(22.6)$ & $116(17.7)$ & \\
\hline$>30 \leq 35$ & $347(54.6)$ & $361(54.9)$ & \\
\hline$>35 \leq 40$ & $133(20.9)$ & $154(23.4)$ & \\
\hline$>40 \leq 45$ & $5(0.8)$ & $20(3.0)$ & \\
\hline$>45 \leq 50$ & $0(0)$ & $1(0.2)$ & \\
\hline Disease duration, yrs & $3.93 \pm 2.24$ & $4.42 \pm 2.48$ & $<0.001$ \\
\hline Primipara & $345(54.2)$ & $404(61.5)$ & 0.01 \\
\hline Twin pregnancy & $6(0.9)$ & $12(1.8)$ & 0.18 \\
\hline \multicolumn{4}{|l|}{ Comorbidity } \\
\hline Hypertension & $8(1.3)$ & $17(2.6)$ & 0.08 \\
\hline Diabetes mellitus & $14(2.2)$ & $19(2.9)$ & 0.43 \\
\hline Uveitis & $88(13.8)$ & $116(17.7)$ & 0.06 \\
\hline IBD & $62(9.7)$ & $76(11.6)$ & 0.29 \\
\hline Psoriasis & $11(1.7)$ & $8(1.2)$ & 0.44 \\
\hline Preterm birth & $14(2.2)$ & $4(0.6)$ & 0.01 \\
\hline Preeclampsia & $4(0.6)$ & $12(2.0)$ & 0.03 \\
\hline \multicolumn{3}{|l|}{ Pattern of treatment } & $<0.001$ \\
\hline No dispensation of medication & $382(60.1)$ & $300(45.7)$ & \\
\hline NSAID alone & $66(10.4)$ & $80(12.2)$ & \\
\hline TNFi, DMARD, or corticosteroids & $188(29.6)$ & $277(42.2)$ & \\
\hline \multicolumn{4}{|c|}{ Drug exposure ${ }^{\dagger}$} \\
\hline NSAID & $196(30.8)$ & $273(41.6)$ & 0.01 \\
\hline Corticosteroids & $108(17)$ & $162(24.7)$ & $<0.001$ \\
\hline DMARD & $93(14.6)$ & $130(19.8)$ & 0.01 \\
\hline TNFi & $53(8.3)$ & $83(12.6)$ & 0.01 \\
\hline
\end{tabular}

Results expressed as the mean $\pm \mathrm{SD}$ or $\mathrm{n}(\%) . * \mathrm{P}$ value was estimated by the $\mathrm{t}$ test for continuous data and the chi-square test or Fisher's exact test for categorical data. ${ }^{\dagger} 12$ months prior to delivery. NSAID: nonsteroidal antiinflammatory drugs; DMARD: disease-modifying antirheumatic drugs; TNFi: tumor necrosis factor inhibitor; IBD: inflammatory bowel disease.

Table 2. Factors associated with cesarean delivery in women with ankylosing spondylitis.

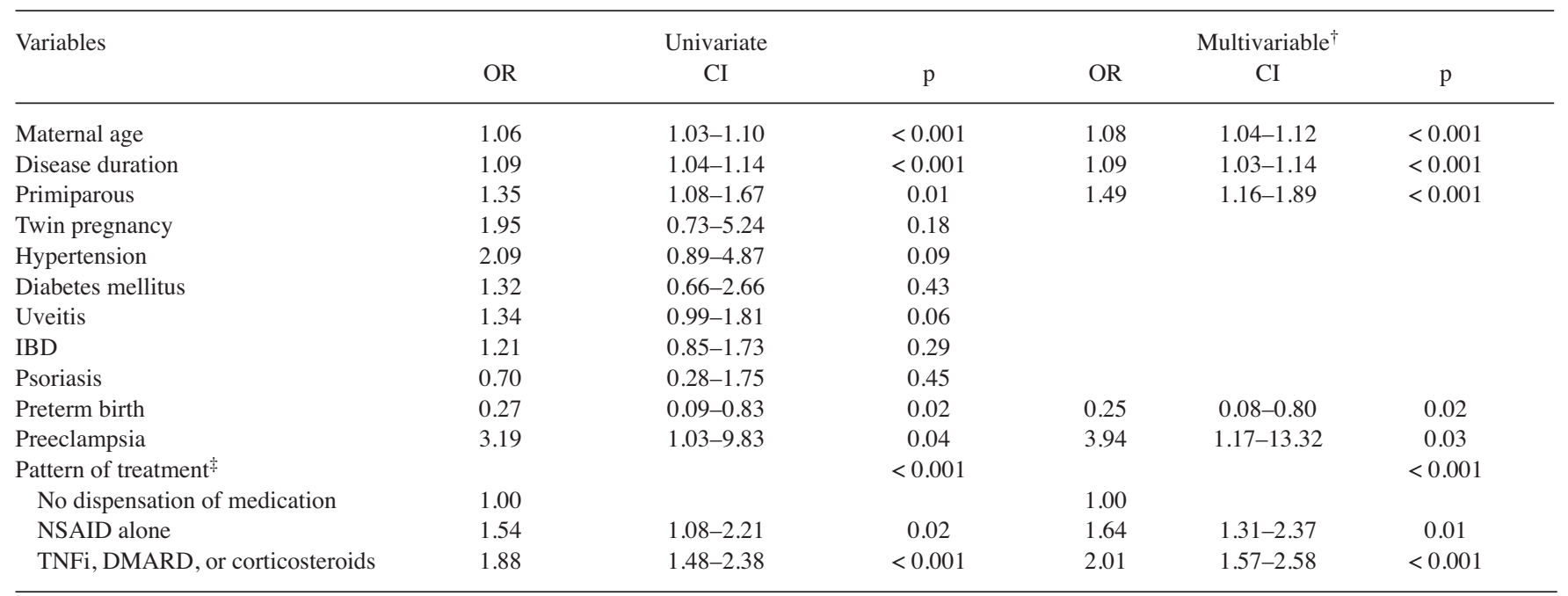

\footnotetext{
$\dagger$ Data were the final results of a backward logistic regression analysis after selecting factors that were significantly associated in the univariate analysis $(\mathrm{p}<0.1) . \stackrel{*}{ } 12$ months prior to delivery. NSAID: nonsteroidal antiinflammatory drugs; DMARD: disease-modifying antirheumatic drugs; TNFi: tumor necrosis factor inhibitor; IBD: inflammatory bowel disease.
} 
of preeclampsia (OR 3.94, 95\% CI 1.17-13.32, p = 0.03) were associated with a higher risk of cesarean delivery. However, preterm birth (OR 0.25 , 95\% CI $0.08-0.80$, $\mathrm{p}=0.02$ ) was associated with a lower probability of cesarean delivery. Compared to no dispensation of medications, NSAID alone and TNFi, DMARD, or corticosteroids were associated with a higher risk of cesarean delivery. Regarding drug exposure, NSAID (OR 1.57, 95\% CI 1.22-2.01, $\mathrm{p}<0.001$ ) or corticosteroids (OR $1.38,95 \%$ CI $1.02-1.85$, $\mathrm{p}=0.03)$ were associated with a higher risk of cesarean delivery (Supplementary Table 2, available with the online version of this article).

Subgroup analysis on primiparas. The results of the subgroup analysis are shown in Table 3. Among the 749 deliveries in primiparas, 404 cases $(53.9 \%)$ were cesarean deliveries. In the univariate analysis, maternal age, disease duration, and pattern of treatment were associated with cesarean delivery. In the multivariable analysis, maternal age (OR 1.14, 95\% CI $1.08-1.19, \mathrm{p}<0.001)$ was associated with a higher risk of cesarean delivery. Compared to no dispensation of medications, NSAID alone (OR 1.89, 95\% CI 1.17-3.04, $\mathrm{p}=0.01$ ) and TNFi, DMARD, or corticosteroids (OR 1.99, 95\% CI $1.44-2.74, \mathrm{p}<0.001$ ) were associated with a higher risk of cesarean delivery. In addition, regarding drug exposure, corticosteroids (OR 1.70, 95\% CI 1.25-2.32, p < 0.001) were associated with a higher risk of cesarean delivery (Supplementary Table 3, available with the online version of this article).

\section{DISCUSSION}

In this Korean population-based study, more than $50 \%$ of the pregnant women with AS underwent cesarean delivery; this rate was higher than in the general population and in women with RA. Older maternal age, longer disease duration, and the presence of preeclampsia were associated with a higher risk of cesarean delivery in women with AS. Further, compared to no dispensation of medications, extensive treatment of AS was associated with a higher risk of cesarean delivery in women with AS.

Previous studies have shown that women with AS showed a higher rate of cesarean delivery than the general population ${ }^{11,12}$. In our present study, the cesarean delivery rate was higher in women with AS than in both women with RA and the general population. Several studies have reported that the risk factors of cesarean delivery in the general population are older maternal age, higher education level, breech presentation, previous cesarean delivery, and maternal request ${ }^{3,4,14}$. Clearly, in our present study, older maternal age was a risk factor for cesarean delivery in women with AS. Further, factors related to AS also contributed to those women choosing cesarean delivery. A longer disease duration of AS was associated with an increased risk of cesarean delivery in the total deliveries but not in the subgroup analysis with primipara. According to our results, the effect of disease duration on the risk of cesarean delivery may be modulated by inherent factors, including prior cesarean delivery. In addition, compared to no dispensation of medications, a higher risk of cesarean delivery was associated with TNFi, DMARD, or corticosteroid, and NSAID alone. In a previous Swedish study, the dispensation of TNFi, DMARD, or corticosteroids was associated with a higher risk of cesarean delivery in women with AS compared to the general population ${ }^{11}$. Interestingly, the analysis of drug exposure revealed that corticosteroids associated with a higher risk of cesarean delivery in women with AS (Supplementary Tables 2 and 3 , available with the online version of this article).

Table 3. Factors associated with cesarean delivery in primiparas among women with ankylosing spondylitis (subgroup analysis).

\begin{tabular}{|c|c|c|c|c|c|c|}
\hline \multirow[t]{2}{*}{ Variables } & \multicolumn{3}{|c|}{ Univariate } & \multicolumn{3}{|c|}{ Multivariable $^{\dagger}$} \\
\hline & OR & $\mathrm{CI}$ & $\mathrm{p}$ & OR & $\mathrm{CI}$ & $\mathrm{p}$ \\
\hline Maternal age & 1.13 & $1.07-1.18$ & $<0.001$ & 1.14 & $1.08-1.19$ & $<0.001$ \\
\hline Twin pregnancy & 0.34 & $0.07-1.76$ & 0.20 & & & \\
\hline Hypertension & 1.20 & $0.38-3.81$ & 0.76 & & & \\
\hline Diabetes mellitus & 1.73 & $0.58-5.10$ & 0.32 & & & \\
\hline Psoriasis & 0.51 & $0.12-2.14$ & 0.36 & & & \\
\hline Preterm birth & 0.12 & $0.02-0.98$ & 0.05 & 0.12 & $0.01-0.97$ & 0.05 \\
\hline Preeclampsia & 1.94 & $0.59-6.36$ & 0.27 & & & \\
\hline Pattern of treatment ${ }^{\ddagger}$ & & & $<0.001$ & & & \\
\hline No dispensation of medication & 1.00 & & & 1.00 & & \\
\hline NSAID alone & 1.69 & $1.07-2.68$ & 0.03 & 1.89 & $1.17-3.04$ & 0.01 \\
\hline
\end{tabular}

$\dagger$ Data were the final results of a backward logistic regression analysis after selecting factors that were significantly associated with the univariate analysis $(\mathrm{p}<0.1) .{ }^{\ddagger} 12$ months prior to delivery. NSAID: nonsteroidal antiinflammatory drugs; DMARD: disease-modifying antirheumatic drugs; TNFi: tumor necrosis factor inhibitor; IBD: inflammatory bowel disease.

Personal non-commercial use only. The Journal of Rheumatology Copyright (C) 2020. All rights reserved. 
Several previous studies have reported that corticosteroids are associated with a higher risk of preterm birth or preeclampsia ${ }^{15,16}$. However, this association is not merely the effect of corticosteroids; rather, the severity of the disease leads to a need for corticosteroid therapy ${ }^{17}$. Corticosteroids are not typically used to treat AS, with the exception of during pregnancy; thus, the use of corticosteroids might reflect an increasing disease activity. Taken together, our results suggest that disease severity or the presence of symptoms requiring more extensive treatment and a longer disease duration before pregnancy affect the choice of delivery method.

Pregnancy-related lower back and/or pelvic girdle pain is common in pregnant women ${ }^{18}$, and patients with AS report higher back pain scores during pregnancy than the general population ${ }^{19}$. Unlike patients with RA, previous studies have reported that most patients with AS experience unchanged or slightly worse disease activity during pregnancy $20,21,22$. Further, aggravation of disease activity is observed in 45-87\% of patients with AS at the 6-month period following delivery ${ }^{23,24}$. In a recent prospective study, pregnant women with AS exhibited a gradual increase of disease activity in the second trimester ${ }^{25}$. Therefore, the presence of symptoms during pregnancy, particularly in the pelvic region, and concerns about pain during delivery or aggravation of disease activity (which might play a role in the preference for cesarean delivery), could be factors behind the higher rate of cesarean delivery in women with AS.

Our present study has some limitations. First, cesarean delivery included both emergent and elective types, and we could not distinguish between them based on the HIRA database. The risk factors for elective and emergent cesarean delivery differ ${ }^{3}$. Thus it is difficult to conclude whether the reason for the high cesarean delivery rate in pregnant women with AS is due to elective cesarean delivery based on maternal request or emergent cesarean delivery based on pregnancy complications. Moreover, although data on disease activity during pregnancy and grade of sacroiliitis were not available, these variables might have contributed to the choice of delivery method. Second, other confounding factors that affect the choice of the delivery method such as socioeconomic status, education level, obesity, smoking history, or baby weight were not identified in the HIRA claims database.

The rate of cesarean delivery was $50.8 \%$ in Korean women with AS, and older maternal age, longer disease duration, and extensive treatment for AS were risk factors for cesarean delivery. The higher cesarean delivery rate observed in women with AS may result from the effects of both maternal age and disease-related factors.

\section{ACKNOWLEDGMENT}

We thank the Korean Health Insurance Review and Assessment Service and the National Health Insurance Service for providing the insurance claims data.
ONLINE SUPPLEMENT

Supplementary material accompanies the online version of this article.

\section{REFERENCES}

1. Betran AP, Torloni MR, Zhang JJ, Gulmezoglu AM; WHO Working Group on Caesarean Section. WHO statement on caesarean section rates. BJOG 2016;123:667-70.

2. Poobalan AS, Aucott LS, Gurung T, Smith WC, Bhattacharya S. Obesity as an independent risk factor for elective and emergency caesarean delivery in nulliparous women-systematic review and meta-analysis of cohort studies. Obes Rev 2009;10:28-35.

3. Wehberg S, Guldberg R, Gradel KO, Kesmodel US, Munk L, Andersson CB, et al. Risk factors and between-hospital variation of caesarean section in Denmark: a cohort study. BMJ Open 2018;8:e19120

4. Rahman M, Shariff AA, Shafie A, Saaid R, Tahir RM. Caesarean delivery and its correlates in northern region of Bangladesh: application of logistic regression and Cox proportional hazard model. J Health Popul Nutr 2015;33:8.

5. OECD. Health at a glance 2017: OECD indicators. Paris: OECD Publishing; 2017 [updated 2017; cited June 4, 2019; accessed January 22, 2020.] Available from: dx.doi.org/10.1787/health_glance-2017-en

6. Chung SH, Seol HJ, Choi YS, Oh SY, Kim A, Bae CW. Changes in the cesarean section rate in Korea (1982-2012) and a review of the associated factors. J Korean Med Sci 2014;29:1341-52.

7. Skomsvoll JF, Ostensen M, Irgens LM, Baste V. Obstetrical and neonatal outcome in pregnant patients with rheumatic disease. Scand J Rheumatol Suppl 1998;107:109-12.

8. Wallenius M, Skomsvoll JF, Irgens LM, Salvesen KA, Nordvag BY, Koldingsnes W, et al. Pregnancy and delivery in women with chronic inflammatory arthritides with a specific focus on first birth. Arthritis Rheum 2011;63:1534-42.

9. de Man YA, Hazes JM, van der Heide H, Willemsen SP, de Groot CJ, Steegers EA, et al. Association of higher rheumatoid arthritis disease activity during pregnancy with lower birth weight: results of a national prospective study. Arthritis Rheum 2009;60:3196-206.

10. Reed SD, Vollan TA, Svec MA. Pregnancy outcomes in women with rheumatoid arthritis in Washington state. Matern Child Health J 2006;10:361-6.

11. Jakobsson GL, Stephansson O, Askling J, Jacobsson LT. Pregnancy outcomes in patients with ankylosing spondylitis: a nationwide register study. Ann Rheum Dis 2016;75:1838-42.

12. Ostensen M, Ostensen H. Ankylosing spondylitis - the female aspect. J Rheumatol 1998;25:120-4.

13. Kim JA, Yoon S, Kim LY, Kim DS. Towards actualizing the value potential of Korea Health Insurance Review and Assessment (HIRA) data as a resource for health research: strengths, limitations, applications, and strategies for optimal use of HIRA data. J Korean Med Sci 2017;32:718-28.

14. Zgheib SM, Kacim M, Kostev K. Prevalence of and risk factors associated with cesarean section in Lebanon - a retrospective study based on a sample of 29,270 women. Women Birth 2017;30: e265-e71.

15. Gur C, Diav-Citrin O, Shechtman S, Arnon J, Ornoy A. Pregnancy outcome after first trimester exposure to corticosteroids: a prospective controlled study. Reprod Toxicol 2004;18:93-101.

16. Palmsten K, Hernandez-Diaz S, Kuriya B, Solomon DH, Setoguchi $\mathrm{S}$. Use of disease-modifying antirheumatic drugs during pregnancy and risk of preeclampsia. Arthritis Care Res 2012;64:1730-8.

17. Bandoli G, Palmsten K, Forbess Smith CJ, Chambers CD. A review of systemic corticosteroid use in pregnancy and the risk of select pregnancy and birth outcomes. Rheum Dis Clin North Am 2017;43:489-502. 
18. Bergstrom C, Persson M, Mogren I. Pregnancy-related low back pain and pelvic girdle pain approximately 14 months after pregnancy - pain status, self-rated health and family situation. BMC Pregnancy Childbirth 2014;14:48.

19. Lui NL, Haroon N, Carty A, Shen H, Cook RJ, Shanmugarajah S, et al. Effect of pregnancy on ankylosing spondylitis: a case-control study. J Rheumatol 2011;38:2442-4.

20. Ostensen M, Husby G. A prospective clinical study of the effect of pregnancy on rheumatoid arthritis and ankylosing spondylitis. Arthritis Rheum 1983;26:1155-9.

21. Ostensen M. The effect of pregnancy on ankylosing spondylitis, psoriatic arthritis, and juvenile rheumatoid arthritis. Am J Reprod Immunol 1992;28:235-7.
22. Ostensen M, Fuhrer L, Mathieu R, Seitz M, Villiger PM. A prospective study of pregnant patients with rheumatoid arthritis and ankylosing spondylitis using validated clinical instruments. Ann Rheum Dis 2004;63:1212-7.

23. Ostensen M, Husby G. Ankylosing spondylitis and pregnancy. Rheum Dis Clin North Am 1989;15:241-54.

24. Timur H, Tokmak A, Turkmen GG, Ali Inal H, Uygur D, Danisman N. Pregnancy outcome in patients with ankylosing spondylitis. J Matern Fetal Neonatal Med 2016;29:2470-4.

25. Ursin K, Lydersen S, Skomsvoll JF, Wallenius M. Disease activity during and after pregnancy in women with axial spondyloarthritis: a prospective multicentre study. Rheumatology 2018;57:1064-71. 